\title{
Graves' Disease Complicated by Ventricular Fibrillation in Three Men Who Were Smokers
}

\author{
Takao Ando, ${ }^{1, \star}$ Tomoko Henmi, ${ }^{2, \star}$ Daisuke Haruta, ${ }^{2}$ Ai Haraguchi, ${ }^{1}$ Ikuko Ueki, \\ Ichiro Horie, ${ }^{1}$ Misa Imaizumi, ${ }^{1}$ Toshiro Usa, ${ }^{1}$ Kouji Maemura, ${ }^{2}$ and Atsushi Kawakami ${ }^{1}$
}

Background: Thyrotoxicosis is known to be associated with sinus tachycardia and supraventricular tachyarrhythmias, but rarely with ventricular fibrillation (Vf), which has only occurred in some patients with hypokalemic periodic paralysis or ischemic heart disease.

Patient Findings: We present three men who were transferred to our hospital with Graves' disease who developed idiopathic Vf. None of them had hypokalemic periodic paralysis or ischemic heart disease but all were smokers. None of other patients with thyrotoxicosis (587 females and 155 males) who were seen at our hospital, in the period during which the three men were seen, had idiopathic Vf. In our three men with thyrotoxicosis and idiopathic Vf, there was no identifiable underlying heart disease. One of the three patients died of hypoxic encephalopathy. The other two men did not have recurrent Vf after their thyroid function normalized.

Summary: These cases and a review of similar cases in the literature imply that improving thyrotoxicosis seems to be effective for treating idiopathic Vf in some patients.

Conclusions: Our findings suggest that thyroid hormone excess might play a direct role in the development of $\mathrm{Vf}$ in susceptible individuals. Our experience with these three patients suggests that smoking men with thyrotoxicosis likely have an increased risk for Vf, even if they do not have other predisposing factors.

\section{Introduction}

G RAVES' DISEASE (GD) is an autoimmune form of hyperThyroidism caused by thyroid-stimulating autoantibodies (1). It is well known that $\sim 90 \%$ of patients with GD are females in their third to fourth decades of life. Increased serum levels of the thyroid hormone cause clinical symptoms including palpitations, hand tremor, hyperhydrosis, and weight loss. It is well established that overt hyperthyroidism induces a hyperdynamic cardiovascular state associated with sinus tachycardia and also increases the prevalence of supraventricular tachyarrhythmias, including atrial premature conduction and atrial fibrillation (2).

The leading causes of sudden cardiac death are coronary artery disease ( $80 \%$ of cases) and cardiomyopathy $(15 \%)$, but $5 \%$ of cases occur in patients with structurally normal hearts (3). Ventricular fibrillation (Vf) is a life-threatening arrhythmia that generally accompanies some form of structural heart disease, most commonly ischemic heart disease associated with a prior myocardial infarction. Less commonly, Vf may be associated with metabolic disorders, drug toxicity, or prolonged or short QT syndrome (3). Vf is a major cause of sudden cardiac death among young people ( $<40$ years) without underlying heart disease (4). Idiopathic $\mathrm{Vf}$ is defined as Vf without any identifiable known cardiac or extracardiac abnormalities responsible for the arrhythmia and accounts for $5 \%-10 \%$ of out-of-hospital survivors of cardiac arrest (4).

It is extremely rare for thyrotoxic patients without underlying cardiac disease to have Vf. Vf attacks have been documented mainly in Asian males suffering from hypokalemic periodic paralysis associated with GD (5-7) and painless thyroiditis (8). In these patients, Vf was likely triggered as a result of a prolonged QT interval produced by their electrolyte disturbance (9). Ischemic heart disease is another cause of $\mathrm{Vf}$ seen in patients with thyrotoxicosis, probably because thyroid hormone is known to increase cardiac oxygen consumption (2). Thus, it is even rarer for thyrotoxic patients not accompanying hypokalemic periodic paralysis to develop Vf without ischemic heart disease (10-14) or with stable coronary disease (15). We present three cases of GD complicated by idiopathic Vf without hypokalemic periodic paralysis or ischemic heart disease. Notably, despite the preponderance of GD in women, all three patients were men, and all three were smokers.

Departments of ${ }^{1}$ Endocrinology and Metabolism and ${ }^{2}$ Cardiovascular Medicine, Nagasaki University Graduate School of Biomedical Sciences, Nagasaki, Japan.

*These two authors equally contributed to this work. 
FIG. 1. Ventricular fibrillation (Vf) seen in Patient 1 (A), Patient 2 (B), and Patient 3 (C). Vf was recorded by an automated external defibrillator in Patient 1 (A) and Patient 3 (C). It should be noted that, in Patient 2, Vf was preceded by frequent premature ventricular complex in the $\mathrm{T}$ wave, recorded by the patient's heart monitor (B).
A

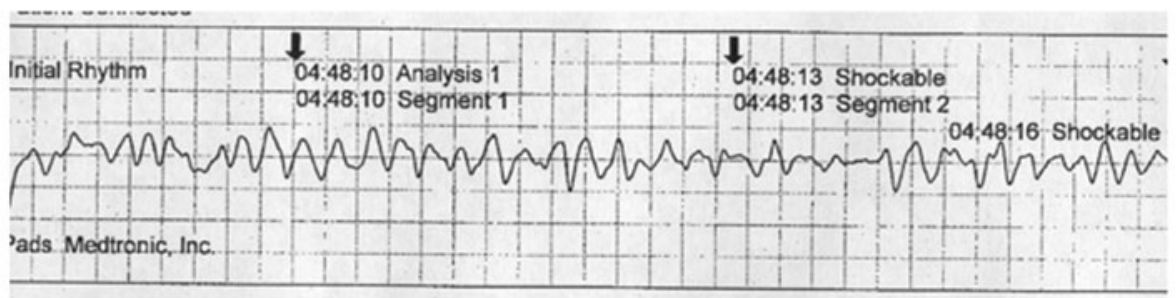

B
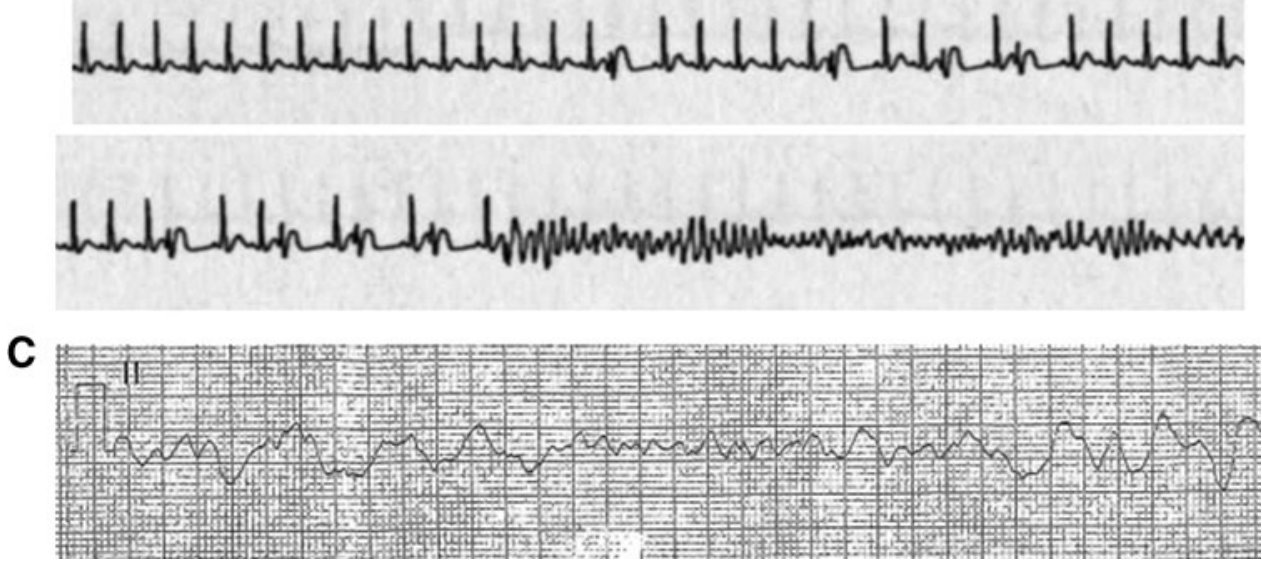

\section{Patients}

\section{Patient 1}

In January 2008, a 43-year-old male smoker was found unconscious in the middle of the night, and when the ambulance came, he was found to be in cardiopulmonary arrest. His Vf (Fig. 1A) was converted to sinus rhythm by using an external defibrillator. He was transferred to our hospital (Na- gasaki University Hospital, Nagasaki, Japan) to undergo hypothermal therapy, because he remained in deep coma and hypoxic encephalopathy was suspected. His medical history was unremarkable except for GD. He had been taking an antithyroid drug irregularly for 8 years and had stopped his medication a couple of weeks before this incident.

His thyroid function tests (Table 1) as determined in our hospital showed high serum levels of free triiodothyronine

Table 1. Clinical Characteristics of Three Patients with Graves' Disease Complicated with Ventricular Fibrillation

\begin{tabular}{|c|c|c|c|}
\hline & Patient 1 & Patient 2 & Patient 3 \\
\hline Age/sex & $43 / \mathrm{M}$ & $39 / \mathrm{M}$ & $45 / \mathrm{M}$ \\
\hline Smoking (Brinkman index) & Yes (750) & Yes (400) & Yes (500) \\
\hline History of syncope & No & No & No \\
\hline Family history of SCD & No & No & No \\
\hline Underlying heart disease & No & No & No \\
\hline Serum K (mEq/L) & 3.2 & 4.8 & 4.2 \\
\hline \multicolumn{4}{|l|}{ Electrocardiogram } \\
\hline Delta wave & No & No & No \\
\hline QTc (s) & 0.36 & 0.32 & 0.32 \\
\hline ST-T change & No & No & No \\
\hline Echocardiography & Unremarkable & Unremarkable & Unremarkable \\
\hline Coronary angiography & Unremarkable & Unremarkable & Unremarkable \\
\hline TSH $(\mu \mathrm{IU} / \mathrm{mL})$ & 0.019 & 0.011 & 0.063 \\
\hline Free T3 (pg/mL) & 9.05 & 12.73 & 4.64 \\
\hline Free T4 (ng/dL) & 2.6 & 8.37 & 3.09 \\
\hline $\mathrm{TRAb}$ & $3.1 \mathrm{IU} / \mathrm{L}$ & $21.5 \%$ & $25.8 \mathrm{IU} / \mathrm{L}$ \\
\hline Treatment for Graves' disease & Radioiodine & Methimazole & Methimazole \\
\hline Outcome & Alive & Alive & Dead \\
\hline Recurrence of $\mathrm{Vf}^{\mathrm{a}}$ & No & No & \\
\hline
\end{tabular}

Reference ranges are 3.5-5.0 mEq/L for serum K, 0.48-5.08 $\mu \mathrm{IU} / \mathrm{mL}$ for TSH, 2.37-3.91 pg/mL for free T3, 0.95-1.57 ng/dL for free T4, and $<1.0 \mathrm{IU} / \mathrm{L}$ and $<15 \%$ for TRAb.

${ }^{a}$ Recurrence of Vf after thyroid function was improved.

QTc, corrected QT interval; SCD, sudden cardiac death; T3, triiodothyronine; T4, thyroxine; TSH, thyrotropin; TRAb, TSH receptor antibodies; $\mathrm{Vf}$, ventricular fibrillation. 
(T3; $9.05 \mathrm{pg} / \mathrm{mL}$; reference range: $2.37-3.91 \mathrm{pg} / \mathrm{mL}$ ) and free thyroxine (T4; $2.6 \mathrm{ng} / \mathrm{dL}$; reference range: $0.95-1.57 \mathrm{ng} / \mathrm{dL}$ ) and suppressed serum thyrotropin (TSH; $0.019 \mu \mathrm{IU} / \mathrm{mL}$; reference range: $0.48-5.08 \mu \mathrm{IU} / \mathrm{mL}$ ). The serum level of TSH receptor antibodies was $3.1 \mathrm{IU} / \mathrm{mL}$ (reference range: $<1.0 \mathrm{IU} /$ L). His thyrotoxicosis was treated using glucocorticoids, potassium iodide, and methimazole, considering his critical general condition.

His electrocardiogram and echocardiography did not suggest any underlying cardiac disorders that might explain his Vf, including aberrant QT interval, ST-T segment, and cardiomyopathies. His blood tests, including assessments of electrolyte, creatinin kinase, and BNP, were unremarkable, although only mild and transient hypokalemia was observed (Table 1). Cardiac sarcoidosis was unlikely because of his normal serum levels of angiotensin-converting enzyme and echocardiography. A few days after admission, he was fully alert and his thyroid function was almost normal after the intensive treatment. His coronary arteries were found to be intact by angiography, including an ergonouvin test to provoke coronary spasm, which was negative. There was no deposition of fat in the right cardiac system on MRI. There was no inducible ventricular tachycardia (VT) or Vf after programmed stimulation at the apex or the outflow tract of the right ventricle. There was no significant change in the ST-T segment, and the electrocardiogram was not Brugada-like, after flecainide infusion. His heart muscle was biopsied, but the microscopic and electromicroscopic examination did not suggest any underlying cardiac disease. Therefore, a diagnosis of idiopathic Vf was made and an implantable cardioverter-defibrillator (ICD) was implanted and his thyroid was ablated with radioiodine therapy. His thyroid function has been well controlled up to the present, and there have been no records of his ICD firing for more than 2 years.

\section{Patient 2}

In March 2003, a 39-year-old male smoker was found unconscious at home just after he awoke. He was found in cardiopulmonary arrest with Vf when the ambulance arrived. Cardioversion was performed, and he was admitted to the local hospital where the cause of Vf could not be identified, although the coronary artery was somewhat spastic after ergonouvin provocation without clinical symptoms of angina or electrocardiogram changes. He was discharged with nitrate tape and advised to quit smoking, and he followed the advice. After 4 uneventful months in which he exhibited normal electrocardiograms, he developed Vf at 2 AM. Cardioversion was performed, and he was admitted to the hospital, but the cause of Vf remained unidentified. He was transferred to our hospital to have an ICD implanted.

When transferred to our hospital, he was semicomatose (JCS-200), because of hypoxic encephalopathy, and was febrile, because of recurrent pulmonary infection. Electrocardiogram and echocardiogram did not suggest any underlying cardiac disorder (Table 1). Three months after the admission, the patient developed incessant Vf (five attacks of Vf a day) (Fig. 1B) on the next day percutaneous endoscopic gastrostomy was performed. Lidocaine and nifekalant as well as cardioversion were used to terminate the $\mathrm{Vf}$, and $400 \mathrm{mg}$ of amiodarone and $30 \mathrm{mg}$ of propranorol were also initiated. He had an ICD implanted a few days after the Vf storm and his level of consciousness improved thereafter.
His thyroid function had never been measured until 2 weeks after starting amiodarone therapy. He was thyrotoxic as indicated by high free $\mathrm{T} 3 \mathrm{of} 6.33 \mathrm{pg} / \mathrm{mL}$, free T4 of $2.62 \mathrm{ng} /$ $\mathrm{dL}$, and suppressed TSH of $<0.005 \mu \mathrm{IU} / \mathrm{mL}$. Methimazole treatment was initiated, although the serum levels of TSH receptor antibodies were not determined. He was transferred to another hospital to continue his rehabilitation.

Nine months after the transfer, the patient visited our hospital. He was ambulant with moderate memory impairment and complained of general fatigue and weight loss. He was thyrotoxic as indicated by free T3 of $15.6 \mathrm{pg} / \mathrm{mL}$, free T4 of $6.97 \mathrm{ng} / \mathrm{dL}$, and TSH of $0.008 \mu \mathrm{IU} / \mathrm{mL}$. His TSH receptor antibodies were positive (TSH binding inhibitory immunoglobulin $21.4 \%$ and thyroid-stimulating antibodies $257 \%$ [reference ranges: $<15 \%$ and $<180 \%$, respectively]) at a time he was not taking amiodarone or methimazole. There was a record of nonsustained VT in ICD. A diagnosis of GD was made, and $30 \mathrm{mg}$ of methimazole was initiated after consulting our endocrine division. We considered that the elevated thyroid function seen at 2 weeks after amiodarone treatment was also due to GD, but not due to amiodoarone-induced thyrotoxicosis. This is because amiodarone-induced thyrotoxocosis has been shown to develop no earlier than a few months after the start of amiodarone treatment (16). Positive TSH receptor antibodies also support the diagnosis of GD. His thyroid function has been euthyroid under the methimazole treatment, and there has been no record of ICD firings for 6 years.

\section{Patient 3}

In February 2010, a 45-year-old male smoker was found unconscious in the middle of the night at home. Vf was recorded (Fig. 1C) and defibrillated by an external defibrillator. He was transferred to the regional hospital where incessant VT and Vf were eventually stabilized with multiple cardioversion procedures. He remained in a deep coma and was transferred to our hospital for hypothermal therapy. He had been treated with methimazole for the previous several months because of his GD, but the methimazole had been temporarily withheld because of druginduced hypothyroidism at 2 weeks before the admission. His thyroid function was assessed in our hospital, and he showed high serum levels of free T3 of $4.64 \mathrm{pg} / \mathrm{mL}$ and free T4 of $3.09 \mathrm{ng} / \mathrm{dL}$ and suppressed TSH of $0.063 \mu \mathrm{IU} /$ $\mathrm{mL}$. Serum levels of TSH receptor antibodies were positive $(25.8 \mathrm{IU} / \mathrm{mL})$. His thyrotoxicosis was treated with methimazole.

His electrocardiogram and echocardiography did not suggest any underlying cardiac disorders. His blood test was unremarkable and the coronary arteries were intact on angiography (Table 1). Therefore, a diagnosis of idiopathic Vf was made. There were no VT or Vf attacks recorded after admission, but he passed away on the 13th day of the admission because of extensive hypoxic encephalopathy.

\section{Retrospective survey of the medical record}

Having seen three patients with GD who developed idiopathic Vf, we reviewed the medical records of the patients with thyrotoxicosis of any cause who were referred to our hospital from March 2002 to March 2010. We were able to find 
Table 2. Clinical Features of Cases of Ventricular Tachycardia and/or Ventricular Fibrillation Associated with Thyrotoxicosis Without Hypokalemic Periodic Paralysis or Ischemic Heart Disease

\begin{tabular}{llll}
\hline Age/sex & Cause of thyrotoxicosis & Type of arrhythmias & Reference \\
\hline 12/F & Graves' disease & Vf & $(10)$ \\
$4 / \mathrm{M}$ & Toxic multinodular goiter & VT & $(11)$ \\
$34 / \mathrm{F}$ & Graves' disease & VT and Vf & $(12)$ \\
$52 / \mathrm{F}$ & Subacute thyroiditis & Incessant VT & $(13)$ \\
$26 / \mathrm{F}$ & Postpartum thyroiditis & Incessant VT & $(14)$ \\
$43 / \mathrm{M}$ & Graves' disease & Vf & Patient 1 \\
$39 / \mathrm{M}$ & Graves' disease & Incessant Vf & Patient 2 \\
$45 / \mathrm{M}$ & Graves' disease & Vf & Patient 3 \\
\hline
\end{tabular}

VT, ventricular tachycardia.

587 female and 155 male patients with thyrotoxicosis, but there were no other patients who developed Vf.

\section{Discussion}

It is extremely rare that thyrotoxicosis is complicated with Vf (2). Vf attacks have been only sporadically documented in patients with thyrotoxic periodic paralysis (5-9) or ischemic heart disease. However, several cases, including ours, involving patients suffering from thyrotoxicosis who developed $\mathrm{Vf}$ and/or VT without concomitant thyrotoxic periodic paralysis or ischemic heart disease have been reported (Table 2). These cases have additional common clinical features such as no family history of sudden cardiac death, no history of syncope attack, and no identifiable causes accounting for VT or Vf, which are thus idiopathic. Interestingly, in four cases found in the literature $(10,11,13,14)$, no ventricular arrhythmias were recorded after the thyroid function was controlled, and therefore, the patients needed only temporary medical treatments for ventricular arrhythmia and did not require the implantation of an ICD. The disappearance of $\mathrm{Vf}$ as a result of the improvement of thyroid function was also seen in Patient 1 and Patient 2 in the present study. Therefore, these findings suggest that thyroid hormone excess, even if it is transient and caused by destructive thyroiditis $(13,14)$, might play a direct role in the development of VT and Vf. Thus, the determination of thyroid function should be considered in patients who develop idiopathic VT and/or Vf.

The three patients reported herein, but no others reported in the literature (Table 2), had common intriguing clinical features. All three patients with GD who developed Vf were smoking men. The smoking habit was shown to be associated with sudden death in an autopsy series studied in our country (17). The constituents of inhaled tobacco are known to damage the cardiovascular system by numerous mechanisms, including endothelial dysfunction, platelet dysfunction, increased coagulation, increased heart rate, blood pressure, increased myocardial oxygen demand, and vasoconstriction. Further, nicotine has been shown to stimulate the sympathetic nerves and markedly elevate serum catecholamine concentration, which is potentially arrhythmogenic (18). Smoking is indeed a significant risk factor for appropriate ICD shocks among patients with heart failure with an ICD (19-21). However, it is not certain whether smoking by itself is a risk for idiopathic Vf. It is known that sudden cardiac death is more common in males (22), and the three smoking men were the only patients who developed Vf among patients who were referred to our hospital because of thyrotoxicosis. As GD is more common in women, thyrotoxicosis including GD may increase the risk of developing life-threatening Vf in smoking men without other predisposing factors.

As the three patients described here were transferred to our hospital and our hospital is a referral center, it would be inappropriate to estimate the prevalence of Vf among patients with thyrotoxicosis or GD. However, it is apparent that the prevalence of $\mathrm{Vf}$ among thyrotoxic patients is notably high in our hospital. It was not clear whether there are unknown factors triggering Vf in our patients or whether the majority of the patients with thyrotoxicosis who develop Vf might have been victims of sudden cardiac death out-of-hospital.

The most important question is how thyrotoxicosis influences the emergence of life-threatening ventricular arrhythmia. It has been shown that several ion transporters present in the cardiac plasma membrane, such as $\mathrm{Na}^{+} / \mathrm{K}^{+}$-ATPase and voltage-gated potassium channels, including Kv1.5, Kv4.2, and $\mathrm{Kv} 4.3$, are upregulated, and the $\mathrm{Na}^{+} / \mathrm{Ca}^{2+}$ exchanger is downregulated by thyroid hormones, thus influencing the electrochemical response of the myocardium (23). However, the influence of thyroid hormones on ventricular depolarization/ repolarization is controversial. Serum TSH levels have been shown to be positively correlated with the corrected QT interval in both men and women in a population-based study (24). Similarly, a QT prolongation was shown in hypothyroidism (25). On the other hand, hyperthyroidism was associated with prolonged QTc intervals $(26,27)$. Although VT and Vf are extremely rare in thyrotoxic patients, even those with hypokalemic periodic paralysis, whether such individuals might possess a genetic predisposition to cardiac arrhythmia in the face of excess thyroid hormones is not known.

We reported three cases of idiopathic Vf complicated with GD. Our experience with these three patients suggests that smoking men with thyrotoxicosis likely have an increased risk for $\mathrm{Vf}$, even if they do not have other predisposing factors. Therefore, the determination of thyroid function should be considered in patients who develop idiopathic VT and/or Vf. More study is needed to address how thyroid hormone excess influences the occurrence of ventricular arrhythmia, and the interaction among smoking, thyrotoxicosis, and ventricular arrhythmias may become apparent in experimental models in the future.

\section{Disclosure Statement}

The authors declare that no competing financial interests exist. 


\section{References}

1. Ando T, Latif R, Davies TF 2005 Thyrotropin receptor antibodies: new insights into their actions and clinical relevance. Best Pract Res Clin Endocrinol Metab 19:33-52.

2. Osman F, Gammage MD, Franklyn JA 2002 Hyperthyroidism and cardiovascular morbidity and mortality. Thyroid 12:483-487.

3. Huikuri HV, Castellanos A, Myerburg RJ 2001 Sudden death due to cardiac arrhythmias. N Engl J Med 345:1473-1482.

4. Tilz RR, Fedele L, Satomi K, Kuck KH, Antz M 2007 Idiopathic ventricular fibrillation. Herz 32:233-239.

5. Boccalandro C, Lopez-Penabad L, Boccalandro F, Lavis V 2003 Ventricular fibrillation in a young Asian man. Lancet 361:1432.

6. Loh KC, Pinheiro L, Ng KS 2005 Thyrotoxic periodic paralysis complicated by near-fatal ventricular arrhythmias. Singapore Med J 46:88-89.

7. Fisher J 1982 Thyrotoxic periodic paralysis with ventricular fibrillation. Arch Intern Med 142:1362-1364.

8. Lee JI, Sohn TS, Son HS, Oh SJ, Kwon HS, Chang SA, Cha BY, Son HY, Lee KW 2009 Thyrotoxic periodic paralysis presenting as polymorphic ventricular tachycardia induced by painless thyroiditis. Thyroid 19:1433-1434.

9. Manoukian MA, Foote JA, Crapo LM 1999 Clinical and metabolic features of thyrotoxic periodic paralysis in 24 episodes. Arch Intern Med 159:601-606.

10. Wolfson B, Smith K 1968 Cardiac arrest following minor surgery in unrecognized thyrotoxicosis: a case report. Anesth Analg 47:672-676.

11. Minegishi Y, Kumada S, Suzuki H, Kusaka H, Shimozawa K, Okaniwa M 1991 Repetitive monomorphic ventricular tachycardia in a 4-year-old boy with toxic multinodular goiter. Acta Paediatr Scand 80:726-731.

12. Jao YT, Chen Y, Lee WH, Tai FT 2004 Thyroid storm and ventricular tachycardia. South Med J 97:604-607.

13. Alper AT, Hasdemir H, Akyol A, Cakmak N 2007 Incessant ventricular tachycardia due to subacute thyroiditis. Int $\mathrm{J}$ Cardiol 116:e22-e24.

14. Vanga S, Patel D, Li H, Lakkireddy D 2008 Incessant right ventricular outflow tract ventricular tachycardia due to subacute postpartum thyroiditis. Europace 10:636-637.

15. Nadkarni PJ, Sharma M, Zinsmeister B, Wartofsky L, Burman KD 2008 Thyrotoxicosis-induced ventricular arrhythmias. Thyroid 18:1111-1114.

16. Eskes SA, Wiersinga WM 2009 Amiodarone and thyroid. Best Pract Res Clin Endocrinol Metab 23:735-751.

17. Owada M, Aizawa Y, Kurihara K, Tanabe N, Aizaki T, Izumi T 1999 Risk factors and triggers of sudden death in the working generation: an autopsy proven case-control study. Tohoku J Exp Med 189:245-258.
18. Adamopoulos D, van de Borne P, Argacha JF 2008 New insights into the sympathetic, endothelial and coronary effects of nicotine. Clin Exp Pharmacol Physiol 35:458-463.

19. Desai H, Aronow WS, Ahn C, Gandhi K, Hussain S, Lai HM, Sharma M, Frishman WH, Cohen M, Sorbera C 2010 Risk factors for appropriate cardioverter-defibrillator shocks, inappropriate cardioverter-defibrillator shocks, and time to mortality in 549 patients with heart failure. Am J Cardiol 105:1336-1338.

20. Goldenberg I, Moss AJ, McNitt S, Zareba W, Daubert JP, Hall WJ, Andrews ML 2006 Cigarette smoking and the risk of supraventricular and ventricular tachyarrhythmias in high-risk cardiac patients with implantable cardioverter defibrillators. J Cardiovasc Electrophysiol 17:931-936.

21. Desai H, Aronow WS, Tsai FS, Ahn C, Lai HM, Amin H, Gandhi K, Frishman WH, Cohen M, Sorbera C 2009 Statins reduce appropriate cardioverter-defibrillator shocks and mortality in patients with heart failure and combined cardiac resynchronization and implantable cardioverterdefibrillator therapy. J Cardiovasc Pharmacol Ther 14:176-179.

22. Kannel WB, Wilson PW, D’Agostino RB, Cobb J 1998 Sudden coronary death in women. Am Heart J 136:205-212.

23. Klein I, Ojamaa K 2001 Thyroid hormone and the cardiovascular system. N Engl J Med 344:501-509.

24. Dorr M, Ruppert J, Robinson DM, Kors JA, Felix SB, Volzke H 2006 The relation of thyroid function and ventricular repolarization: decreased serum thyrotropin levels are associated with short rate-adjusted QT intervals. J Clin Endocrinol Metab 91:4938-4942.

25. Fazio S, Biondi B, Lupoli G, Cittadini A, Santomauro M, Tommaselli AP, Lombardi G, Sacca L 1992 Evaluation, by noninvasive methods, of the effects of acute loss of thyroid hormones on the heart. Angiology 43:287-293.

26. Colzani RM, Emdin M, Conforti F, Passino C, Scarlattini M, Iervasi G 2001 Hyperthyroidism is associated with lengthening of ventricular repolarization. Clin Endocrinol (Oxf) 55:27-32.

27. Owecki M, Michalak A, Nikisch E, Sowinski J 2006 Prolonged ventricular repolarization measured by corrected QT interval (QTc) in subclinical hyperthyroidism. Horm Metab Res 38:44-47.

Address correspondence to: Takao Ando, M.D., Ph.D.

Department of Endocrinology and Metabolism Nagasaki University Graduate School of Biomedical Sciences 1-7-1 Sakamoto Nagasaki 852-8501 Japan

E-mail: takaoando@gmail.com 